\title{
Transient Crossed Aphasia: a Case Study with SPEGT
}

\author{
JOSÉ M. FERRO, ${ }^{1,2}$ GUILHERMINA CANTINHO ${ }^{3}$ and ÉLIA BAETA ${ }^{1,2}$ \\ ${ }^{1}$ Department of Neurology and ${ }^{2}$ Language Research Laboratory, Centro de Estudos Egas Moniz, \\ ${ }^{3}$ Department of Nuclear Medicine, Hospital de Santa Maria, University of Lisbon, Portugal \\ Correspondence and reprints request: 7. M. Ferro, Centro de Estudos Egas Moniz, Hospital de Santa \\ Maria, 1600 Lisboa, Portugal.
}

\begin{abstract}
A 45-year-old right-handed man developed "crossed" anomic aphasia, neglect and inaccurate reaching of his left arm in both hemispaces due to a right parietal haemorrhage. Aphasia cleared in a few days. SPECT failed to demonstrate crossed left hemispheric diaschisis. This report emphasizes the importance of evaluating patients in the acute stage and of coupling "static" and "functional" imaging methods when studying brain-behavior relationships.
\end{abstract}

\section{Introduction}

To diagnose crossed aphasia (aphasia following right hemispheric lesion in a right-handed subject) one has to exclude by appropriate neuroimaging methods or autopsy concomitant left hemispheric lesions (Joannette et al., 1982; Basso et al., 1985; Castro-Caldas et al., 1987). However the role of crossed diashisis (Feeney and Baron, 1986), i.e. of a functional disturbance of the contralateral areas of the left hemisphere, following infarction of the "mirror areas" of the right hemisphere, has not been studied, with the exception of the two cases reported by Perani and associates (Perani et al., 1988), in whom SPECT failed to detect any left hemispheric diaschisis.

\section{Case Report}

A 45-year-old hypertensive male had a sudden onset of frontal headache, left-sided weakness and speech disturbance. On admission blood pressure was $170-100 \mathrm{~mm} \mathrm{Hg}$. He was right handed for writing and all daily activities and he had no left-handed relatives. Speech was fluent with anomia and semantic paraphasias. He was severely disturbed when naming visually presented objects, but could repeat words and sentences and follow one-step commands. He had left-sided neglect and was anosognosic for his left hemiparesis. A CT scan showed a right parietal haematoma, visible on three CT cuts, involving the angular gyrus subcortically and reaching the cortex of the superior parietal lobe (Fig. 1). Two old right hemispheric lesions were also visible: a slit lenticular-external capsule hypodensity, probably corresponding to an old haemorrhage, and a thalamic lacune. 

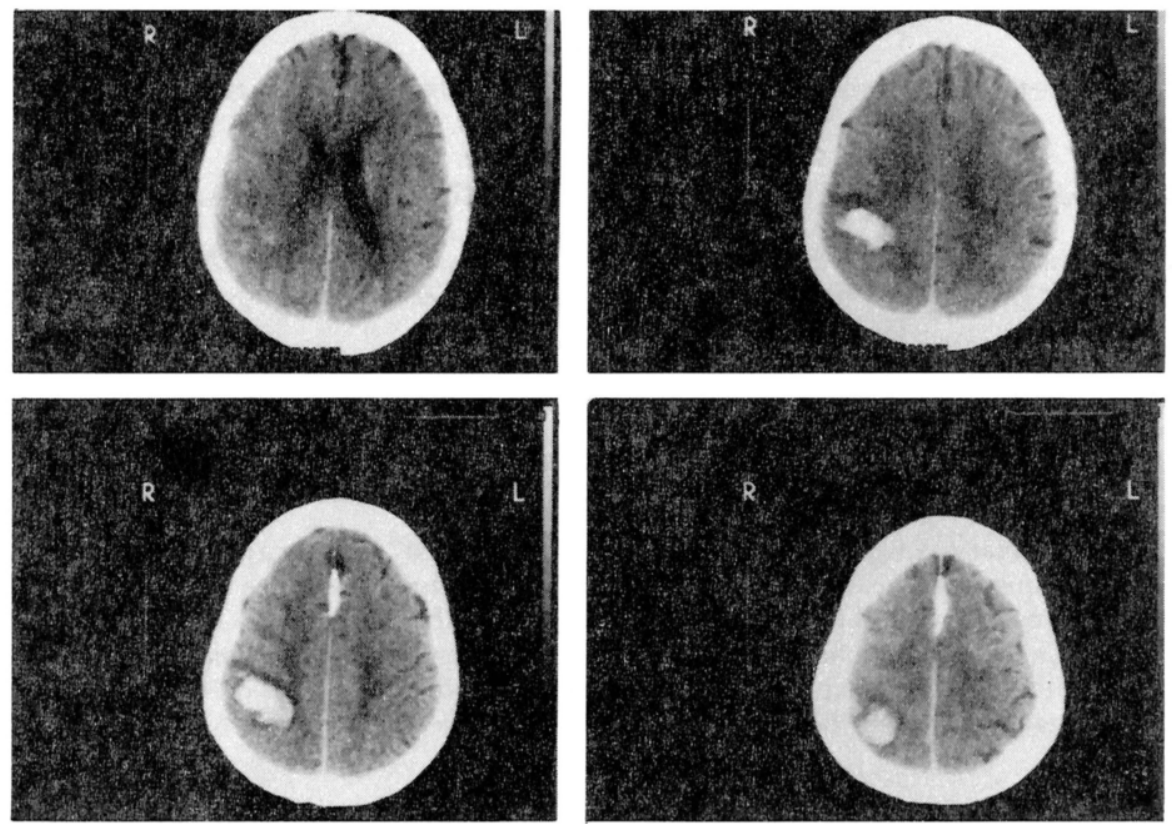

FIg. 1. CT scan: right parietal haematoma, involving the white matter deep to the angular gyrus and the superior parietal lobe.

Both of these lesions had not produced clinical signs. Two days later his limb weakness was greatly improved, but the patient showed a marked disturbance on reaching targets with the right hand in both hemispaces. On examination, he still had a transcortical sensory aphasia: speech was fluent with word finding and semantic disturbances. He named $5 / 5$ objects, $0 / 2$ body parts, $1 / 3$ colours. He could follow $3 / 4$ one-step commands but none of 3 two-step orders. He repeated 10/10 words and 2/2 sentences. He demonstrated a striking neglect hemialexia. For instance on the sentence $O \mathcal{F O} \tilde{A O}$ FOI PARA A PRAIA (John went to the beach) he read FOI PARA A PRAIA. His writing was normal, although he only used the left half of the page. He had no bucco-facial or limb apraxia, but was severely impaired on drawing spontaneously or on copying, demonstrating severe left hemispatial neglect. On a cancellation test he crossed 6/7 of the right lines, and none of the central or left-sided ones.

He was treated with bedrest, nifedipine and propranolol and his condition rapidly improved. Digital intra-arterial anteriography was normal.

Neuropsychological testing was repeated 1 week later. On a standardized aphasia battery (Ferro, 1986) he had minor speech output impairment, mainly due to word finding pauses. He had a $100 \%$ performance on naming, comprehension and repetition tests. However, on a 22 item abbreviated form of the Token test, he had a rather low score of 12 (lower than mean minus $3 \mathrm{SD}$, for his age and education). Reading, writing and 
praxis were normal. He had a borderline performance (39 points) on Benton's Facial Recognition Test. Drawing was mildly impaired (10/15), showing asymmetric arrangement of details. Performance on the BentonFogel three-dimensional constructional praxis test was poor. On a comprehensive neglect testing battery (Ferro et al., 1987) he had partial visual $(5 / 10$ on the left hemifield) and tactile extinction (7/10) on bilateral simultaneous stimulation, but cancellation and line bissection failed to reveal hemispatial neglect. The right hand reaching disturbance was no longer present.

A repeat CT scan performed on the same day showed a resolving superior parietal haematoma, visible on two CT cuts. SPECT was performed on the same day using HMPAO, labelled with $99 \mathrm{~m} \mathrm{Tc}$, in a $400 \mathrm{AC}$ GE Gamma camera. $740 \mathrm{MBq}$ (20mCi) tomography data were collected using the following parameters: 360 elliptical rotation, $128 \times 128$ matrix, 64 projection, pixel size of $0.31 \mathrm{~cm}$ and accumulating time $30 \mathrm{~s}$ projection. Data were processed using the back projection methodology with a RampHanning filter. A relative quantification of cerebral blood flow rCBF (mean and $\mathrm{SD}$ ) was done by measuring $\mathrm{rCBF}$ on symmetrical regions of interest (ROI). ROI boxes ( $4 \times 4$ pixel size) were placed on two subcortical areas, anterior (lenticular) and posterior (thalamic) and three cortical zones, anterior (frontal), central (sylvian), posterior (temporo-parietal) rCBF was measured on three axial slices ( 2 pixels thick). A semiquantitative assessment of $\mathrm{rCBF}$ was calculated by means of a relative perfusion index between the two hemispheres, index values between 0.90 and 1.10 being considered normal. Five index values for each slice were calculated by the ratio between symmetrical cortical (anterior, central, posterior) and subcortical (anterior, posterior) zones. Hypoperfusion was found only in the right postrolandic region and in an adjacent posterior subcortical (thalamic) area (Table 1, Fig. 2).

\section{Discussion}

This patient was unequivocally right handed and developed aphasia and several "parietal" disturbances (constructional apraxia, inaccurate reaching, neglect) after a right parietal haemorrhage. There were no CT visible lesions in the left hemisphere. Although he had two old (thalamic and

TABLE 1. SPECT: interhemispheric perfusion index

\begin{tabular}{llllll}
\hline \multirow{2}{*}{ Area } & & \multicolumn{4}{c}{ Left right Perfusion Index } \\
& & Cut 1 & Cut 2 & Cut 3 & Cut 4 \\
\hline Cortical & anterior & 0.91 & 0.96 & 1.0 & 0.98 \\
& middle & 1.01 & 1.05 & 1.0 & 0.97 \\
& posterior & 0.91 & $1 \cdot 18^{*}$ & $1.30^{*}$ & $1.30^{*}$ \\
Subcortical & anterior & 1.06 & 0.99 & 0.95 & - \\
& posterior & 1.05 & 1.01 & $1.10^{*}$ & - \\
\hline
\end{tabular}

* Significant assymmetries 


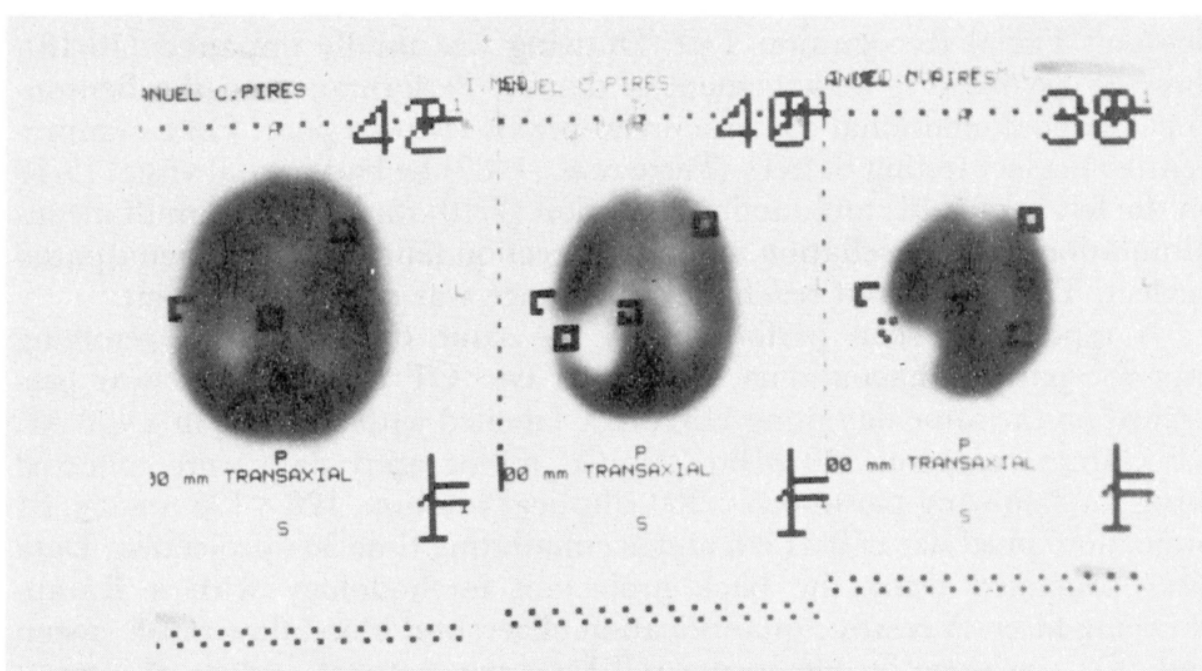

FIg. 2. 99 HMPAO SPECT: three consecutive transaxial slices demonstrating focal hypoperfusion on the right temporo parietal region.

lenticular) right subcortical lesions, aphasia appeared suddenly and improved quickly in relation to the onset of the right haematoma and its reabsorption. Briefly, this patient met all the criteria for crossed aphasia. The possibility of the language disturbance being caused by crossed diaschisis of the left hemisphere was also excluded because SPECT showed decreased cerebral perfusion only on the right hemisphere. Perani and associates (1988) studied two cases of crossed aphasia with SPECT and demonstrated a relationship between the presence and severity of aphasia and decreased $\mathrm{CBF}$ on right hemispheric structures. In their case 2 a subcortical infarct was associated with profound cortical hypoperfusion. In our patient the area of reduced CBF extended beyond the superior parietal lobe where CT visualized the haematoma, thus explaining the comprehension defect that persisted on the Token Test even after aphasia had almost cleared. These observations show that it is inappropriate to consider "anomalous", those cases of crossed aphasia whose aphasia profiles are atypical for their lesion location (Alexander et al., 1989), without performing functional (SPECT, PET) studies.

Recently, Alexander et al. (1989) proposed two types of crossed aphasia: mirror image and "anomalous". The case we report belongs to the "mirror image" group, because his aphasia profile is comparable to that one would expect from a symmetrically placed left hemisphere lesion. The language disturbance lasted less than 10 days, indicating that some "mirror" crossed aphasias can have exceptional recoveries, and that this should be taken into consideration when constructing hypotheses related to crossed aphasia (Alexander et al., 1989). 


\section{Acknowledgement}

The authors thank Dr Paula Esperança, Alice Levy and F. Godinho for their contribution in the study of this patient.

\section{References}

Alexander, M. P., Fischette, M. R. and Fisher, R. R. S. (1989). Crossed aphasias can be mirror image or anomalous. Brain, 112, 953-973.

Basso, A., Capitani, E., Laicona, M. and Zanobio, M. E. (1985). Crossed aphasia: one or more syndromes? Cortex, 21, 25-45.

Castro Caldas, A., Confraria, A. and Poppe, P. (1987). Non-verbal disturbances in crossed aphasia. Aphasiology, 1, 403-413.

Feeney, D. M. and Baron, J. C. (1986). Diaschisis. Cortex, 17, 817-830.

Ferro, J. M. (1986). "Neurologia do Comportamento". Faculdade de Medicina de Lisboa, Lisboa.

Ferro, J. M., Kertesz, A. and Black, S. (1987). Subcortical neglect: anatomy, quantitation and recovery. Neurology, 37, 1487-1492.

Joanette, Y., Puel, M., Nespoulous, J. L., Rasciol, A. and Lecours, A. R. (1982). Aphasie croissée chez les droitiers. I Revue de la littérature. Revue Neurologique, 138, 575-586.

Perani, D., Papagno, C., Cappa, S., Gerundini, P., Fazio, F. (1988). Crossed aphasia: functional studies with single photon emission computerized tomography. Cortex, 24, 171-178. 


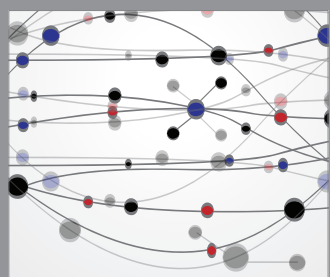

The Scientific World Journal
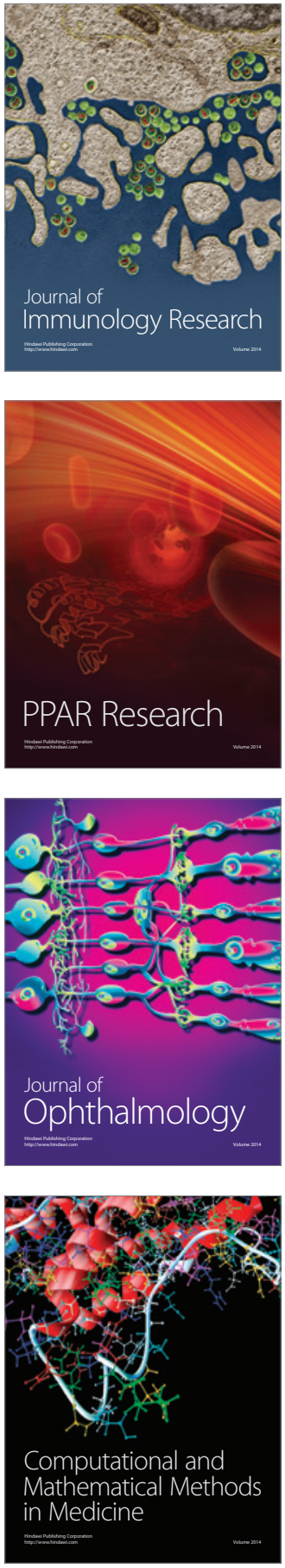

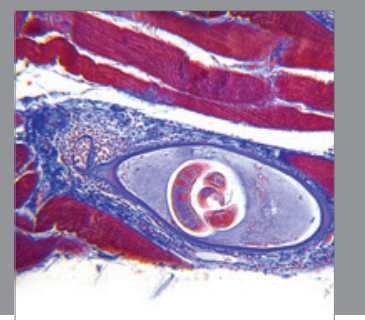

Gastroenterology

Research and Practice
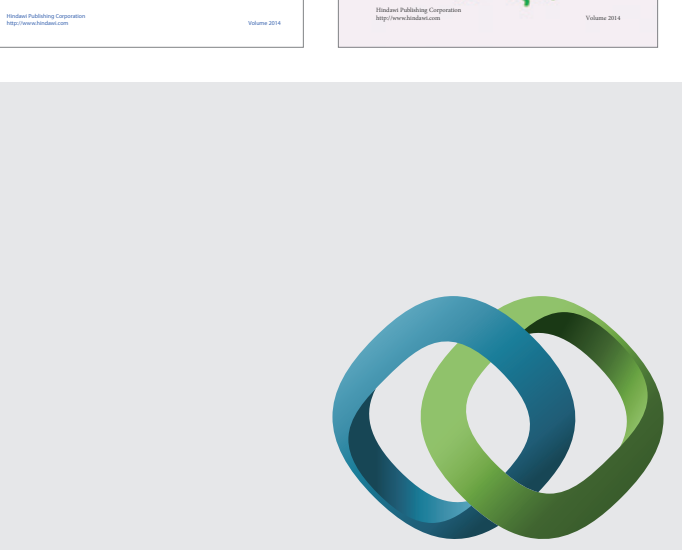

\section{Hindawi}

Submit your manuscripts at

http://www.hindawi.com
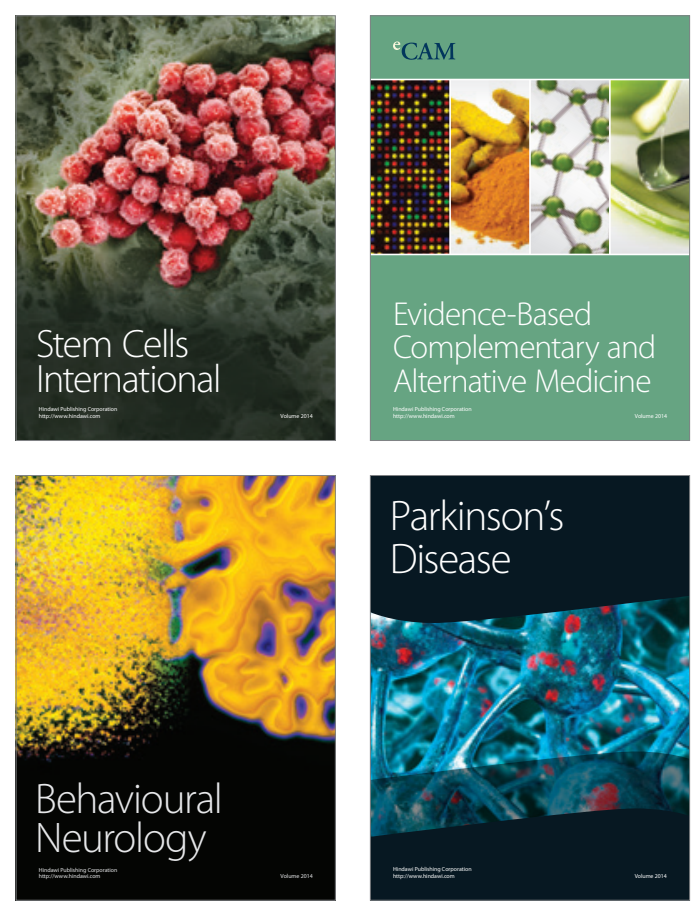

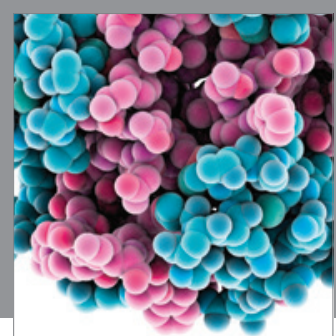

Journal of
Diabetes Research

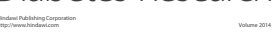

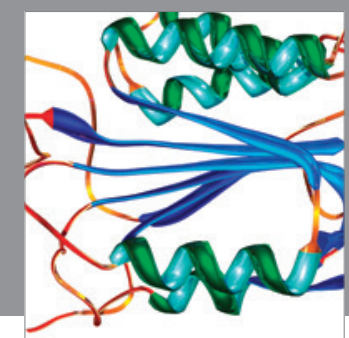

Disease Markers
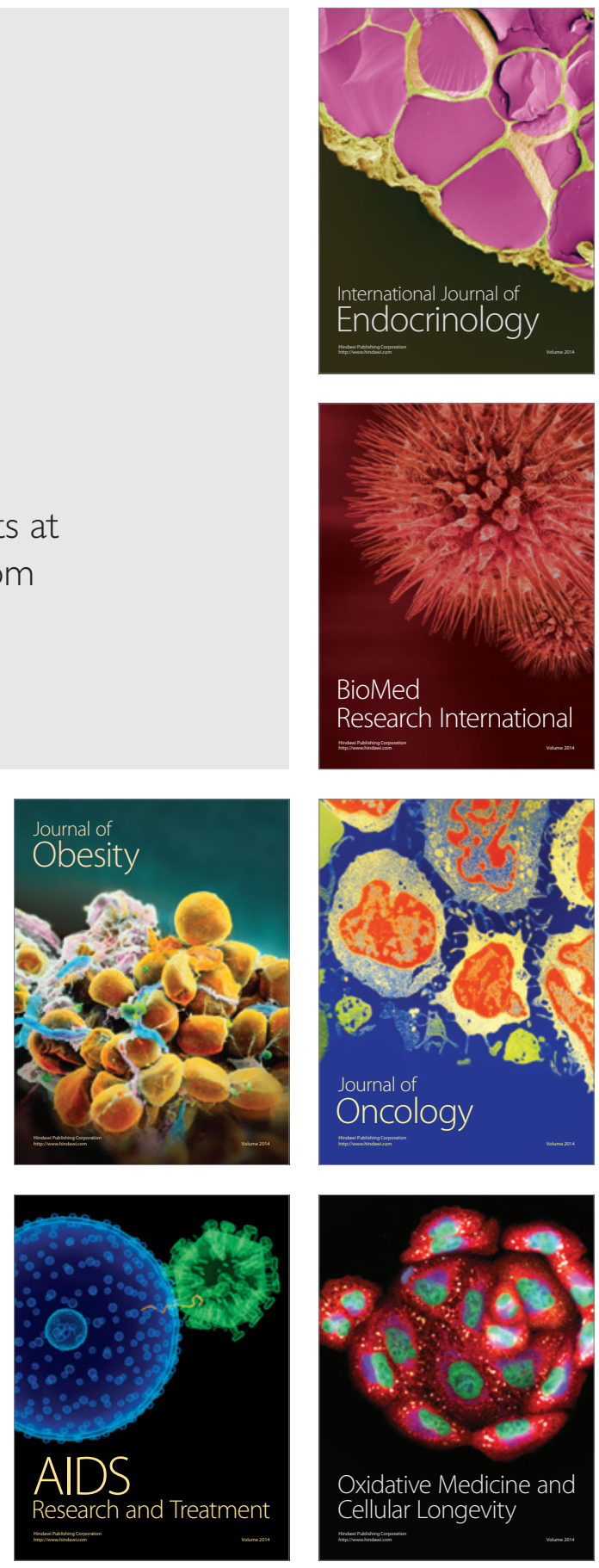